\title{
Is There a Scientific Basis for Disaster Health and Medicine?
}

\author{
Samuel J. Stratton, MD, MPH
}

Is there a need for scientific support for disaster health and medicine? It seems that there is an accepted disaster health and medicine knowledge base that is based largely on opinions of those who have deemed themselves expert in disaster health, or based on descriptive experiences of some during disaster deployments. While opinion and experience may be methods for determining standards for health and medicine in disasters, scientific exploration is the basis for forming the knowledge base of a respected and effective domain in the various areas of health and medicine. In a sarcastic view, if opinion and singular experience were an acceptable basis for establishing knowledge, the Western civilizations would still consider the earth the center of the universe.

At present, disaster health and medical science continues to develop slowly, despite three decades of recognition by early pioneers in the disaster medicine field of the need for application of scientific principles. Certainly, attaining "pure" disaster science with randomized controlled trials to show causal effects is likely not attainable because of the need to prospectively control study variables. Because of the nature of disaster events, most variables can rarely be controlled. On the other hand, epidemiologic and qualitative methods that provide associations among variables are applicable to disaster research and provide effective working science knowledge for the field. Additionally, disaster medical research is particularly appropriate for the developing field of simulation research. Simulation research allows for development of disaster event models that can be tested against actual events as they occur and can provide for control of important variables such that disaster effects can be predicted.

Disaster health and medical research is developing, and it is worth evaluating where it stands at this time. As with many medical journals, Prehospital and Disaster Medicine uses a standardized scoring system for peer review of scientific submissions. Additionally, the types of submissions have been logged to determine the most frequent research methodologies for papers submitted to the journal. During the past year (June 2013 through May 2014) both the peer review scoring and the research methodology for submissions have shown trends that may illustrate the current state of disaster health and medical research. Of course there are many journals that publish disaster health and medical material, so the external validity of the Prehospital and Disaster Medicine experience is not known.

Among research methodologies, the most common type of original research submission to Prehospital and Disaster Medicine is survey based. In the past year, $64 \%$ of all submissions were primarily survey based. This includes papers that were stated to be of mixed research methodology. Most commonly, reported mixed-method papers that used surveys used the surveys as the primary means for making a study conclusion. Following survey-based research; descriptive case series are most common for submission (23\% of all original research submissions). Case series are a common method for reporting research in the area of mass gatherings and multi-casualty incidents. Combining survey and descriptive case series methodologies accounts for $87 \%$ of all original research category submissions to the Journal. Descriptive and analytical database studies accounted for $7 \%$ of original research submissions. Database studies were separated from case series based on the use of large, validated databases for the research as opposed to logged episodes at a specific event(s). Cohort population studies accounted for another $2 \%$ of submissions. The remaining $4 \%$ of submissions included simulation-based research (including modeling), descriptive interventional studies, and comparative analysis of similar events.

Considering the above submission statistics for the Journal, it appears that a current focus of high-quality survey and case series research is likely to advance disaster health and medical research at the current time. Further, development of more robust research methodology, particularly using epidemiologic and simulation techniques, will likely be means for future advancement of the knowledge base for disaster health and medicine.

Further internal statistics for Prehospital and Disaster Medicine are those for selection of submissions for publication. While many journals publish and rank themselves based on rejection rates for submissions, it is the intent of Prehospital and Disaster Medicine to publish as many papers that contribute to the knowledge base as available print space will allow. Cost of publishing print editions of the Journal limits the number of pages available and therefore the ability to select all submissions for publication.

Statistics for selection of submissions for publication reveal that often papers are not selected for five primary reasons (Table 1). Most common problems that lead to a paper being rejected center around vagueness of the study objective or hypothesis and management of data. Most important is the issue of researcher bias and lack of objectivity. True scientific research is based on the ability to put aside biases and objectively seek the answer to a defined question of knowledge. Often papers cannot be selected because of methodological errors such as too few study subjects or lack of definition of data elements. Particularly problematic is selection bias that may occur in survey-based research with lack of survey response that leads to large margins of error in results. Studies that are designed with attention to limiting any form of bias are most likely to be selected for publication in the Journal.

It is hoped that the above information is helpful in describing the state of the research in the field and will serve as a guide for those active in disaster health and medical research. Prehospital and Disaster Medicine takes no pride in limiting submitted manuscripts for publication and rather will make every endeavor to publish well-designed research that adds to the disaster health and medicine knowledge base. It is the philosophy of the Journal to publish well-performed research, no matter how 
1. Poorly-defined, vague, or rambling study objective.

2. Study population too small for valid conclusion:

A. Large margin of error

B. Overlap of data precision measurements (confidence intervals, inter-quartile ranges).

3. Lack of recognition of variables (confounders) that may affect results.

4. Lack of objectivity and bias in study design such that discussion and conclusions are not supported by the study data, or data is collected with bias.

5. Non-valid survey or study database with failure to define important data elements.

6. Conclusion does not reflect study data, over-interpretation of study data.

7. Study data reporting errors with inconsistent reporting of data in paper text, tables, and charts.

8. Lack of Research Ethics Committee (human subjects committee, institutional review board) review or exemption when appropriate.

9. Methodological flaws in management and analysis of data:
A. Mixing populations that are inconsistent with each other
B. Failure to select a study group that reflects the population of interest
C. Applying statistical tests inappropriately for the data type
D. Misinterpretations of study data in results and discussion sections.

10. Data dredging - examining data without a defined study objective to arrive at any presumed conclusion that can be put forth.

(c) 2014 Prehospital and Disaster Medicine

Table 1. Most Common Reasons for Inability to Accept Submissions for Publication in Prehospital and Disaster Medicine (June 2013-May 2014), Listed in Order of Frequency

obscure the topic. This philosophy varies from that of many journals in that it limits the ability for Prehospital and Disaster Medicine to compete with other journals for citation numbers and popularity measures. However, the goal of the editorial board of this Journal is to encourage valid science and knowledge development; it is hoped that this approach allows Prehospital and Disaster Medicine to make valuable contributions to the health and medicine community.

doi:10.1017/S1049023X14000582 